\title{
Variability in Data Streams
}

\author{
David Felber \\ UCLA \\ dvfelber@ucla.edu
}

\author{
Rafail Ostrovsky \\ UCLA \\ rafail@cs.ucla.edu
}

\begin{abstract}
We consider the problem of tracking with small relative error an integer function $f(n)$ defined by a distributed update stream $f^{\prime}(n)$ in the distributed monitoring model. In this model, there are $k$ sites over which the updates $f^{\prime}(n)$ are distributed, and they must communicate with a central coordinator to maintain an estimate of $f(n)$.

Existing streaming algorithms with worst-case guarantees for this problem assume $f(n)$ to be monotone; there are very large lower bounds on the space requirements for summarizing a distributed non-monotonic stream, often linear in the size $n$ of the stream. However, the input streams obtaining these lower bounds are highly variable, making relatively large jumps from one timestep to the next; in practice, the impact on $f(n)$ of any single update $f^{\prime}(n)$ is usually small. What has heretofore been lacking is a framework for nonmonotonic streams that admits algorithms whose worst-case performance is as good as existing algorithms for monotone streams and degrades gracefully for non-monotonic streams as those streams vary more quickly.

In this paper we propose such a framework. We introduce a stream parameter, the "variability" $v$, deriving its definition in a way that shows it to be a natural parameter to consider for non-monotonic streams. It is also a useful parameter. From a theoretical perspective, we can adapt existing algorithms for monotone streams to work for non-monotonic streams, with only minor modifications, in such a way that they reduce to the monotone case when the stream happens to be monotone, and in such a way that we can refine the worst-case communication bounds from $\Theta(n)$ to $\tilde{O}(v)$. From a practical perspective, we demonstrate that $v$ can be small in practice by proving that $v$ is $O(\log f(n))$ for monotone streams and $o(n)$ for streams that are "nearly" monotone or that are generated by random walks. We expect $v$ to be $o(n)$ for many other interesting input classes as well.
\end{abstract}

Permission to make digital or hard copies of all or part of this work for personal or classroom use is granted without fee provided that copies are not made or distributed for profit or commercial advantage and that copies bear this notice and the full citation on the first page. Copyrights for components of this work owned by others than ACM must be honored. Abstracting with credit is permitted. To copy otherwise, or republish, to post on servers or to redistribute to lists, requires prior specific permission and/or a fee. Request permissions from permissions@ acm.org.

PODS '16, June 26-July 1, 2016, San Francisco, CA, USA.

(c) 2016 ACM. ISBN 978-1-4503-4191-2/16/06 . .\$15.00

DOI: http://dx.doi.org/10.1145/2902251.2902277

\section{CCS Concepts}

- Theory of computation $\rightarrow$ Data structures and algorithms for data management; Sketching and sampling; Lower bounds and information complexity; Streaming models; •Information systems $\rightarrow$ Data streams;

\section{Keywords}

data stream; non-monotonic stream; distributed monitoring model; tracking; counting; variability

\section{INTRODUCTION}

In the distributed monitoring model, there is a single central monitor and several $(k)$ observers. The observers receive data and communicate with the monitor, and the goal is to maintain at the monitor a summary of the data received at the observers while minimizing the communication between them.

This model was introduced by Cormode, Muthukrishnan, and $\mathrm{Yi}[4,5]$ with the motivating application of minimizing radio energy usage in sensor networks, but can be applied to other distributed applications like determining network traffic patterns. Since the monitor can retain all messages received, algorithms in the model can be used to answer historical queries too, making the model useful for auditing changes to and verifying the integrity of time-varying datasets.

The distributed monitoring model has also yielded several theoretical results. These include algorithms and lower bounds for tracking total count $[4,5,10,11]$, frequency moments $[4,5,8,14,15]$, item frequencies $[8,14,15,16,17]$, quantiles $[8,14,15,16,17]$, and entropy $[1,14,15]$ to small relative error.

However, nearly all of the upper bounds assumed that data is only inserted and never deleted. This assumption is unfortunate because in the standard turnstile streaming model, all of these problems have similar algorithms that permit both insertions and deletions. In general, this unfortunate assumption is unavoidable; existing lower bounds for the distributed model [1] demonstrate that it is not possible to track even the total item count in small space when data is permitted to be deleted.

That said, when restrictions are placed on the types of allowable input, the lower bounds evaporate, and very nice upper bounds exist. Tao, Yi, Sheng, Pei, and Li [13] developed algorithms for the problem of summarizing the order statistics history of a dataset $D$ over an insertion/deletion stream of size $n$, which has an $\Omega(n)$-bit lower bound in general; how- 
ever, they performed an interesting analysis that yielded online and offline upper bounds proportional to $\sum_{t=1}^{n} 1 /|D(t)|$, with a nearly matching lower bound. A year or two later, Liu, Radunović, and Vojnović $[10,11]$ considered the problem of tracking $|D|$ under random inputs; for general inputs, there is an $\Omega(n)$-bit lower bound, but Liu et. al. obtained (among other results) expected communication costs proportional to $\sqrt{n} \log n$ when the insertion/deletion pattern is the result of fair coin flips.

In fact, the pessimistic lower bounds for the general case can occur only when the input stream is such that the quantity being tracked is forced to vary quickly. In the problems considered by Tao et. al. and Liu et. al., this occurs when $|D|$ is usually small. These two groups avoid this problem in two different ways: Tao et. al. provide an analysis that yields a worst-case upper bound that is small when $|D|$ is usually large, and Liu et. al. consider input classes for which $|D|$ is usually large in expectation.

\section{Our contributions.}

In this paper we propose a framework that extends the analysis of Tao et. al. to the distributed monitoring model and that permits worst-case analysis that can be specialized for random input classes considered by Liu et. al. In so doing, we explain the intuition behind the factor of $\sum_{t=1}^{n} 1 /|D(t)|$ in the bounds of Tao et. al. and how we can separate the different sources of randomness that appear in the algorithms of Liu et. al. to obtain worst-case bounds for the random input classes we also consider.

In the next section we derive a stream parameter, the variability $v$, that is a function of the distributed update stream $f^{\prime}(n)$. We prove that $v$ is $O(\log f(n))$ for monotone streams and $o(n)$ for streams that are "nearly" monotone or that are generated by random walks, and find that the bounds of Tao et. al. and Liu et. al. are stated nicely in terms of $v$. In section 3 we combine ideas from the upper bounds of Tao et. al. [13] with the existing distributed counting algorithms of Cormode et. al. [4, 5] and Huang, Yi, and Zhang [8] to obtain upper bounds for distributed counting that are proportional to $v$. In section 4 we show that our dependence on $v$ is essentially necessary by developing deterministic and randomized space+communication lower bounds that hold even when $v$ is small. We round out the piece in section 5 with a discussion of the suitability of variability as a general framework, in which we extend the ideas of section 3 to the problems of distributed tracking of item frequencies and of tracking general aggregates when $k=1$.

But before we jump into the derivation of variability, we define our problem formally and abstract away unessential details.

\section{Problem definition.}

The problem is that of tracking at the coordinator an integer function $f(n)$ defined by an update stream $f^{\prime}(n)$ that arrives online at the sites. Time occurs in discrete steps; to be definite, the first timestep is 1 , and we define $f(0)=0$ unless stated otherwise. At each new current time $n$ the value $f^{\prime}(n)=f(n)-f(n-1)$ appears at a single site $i(n)$.

There is an error parameter $\varepsilon$ that is specified at the start. The requirement is that, after each timestep $n$, the coordinator must have an estimate $\hat{f}(n)$ for $f(n)$ that is usually good. In particular, for deterministic algorithms we require that $\forall n,|f(n)-\hat{f}(n)| \leq \varepsilon f(n)$, and for randomized algorithms we require that $\forall n, P(|f(n)-\hat{f}(n)| \leq \varepsilon f(n)) \geq 2 / 3$.

\section{VARIABILITY}

In the original distributed monitoring paper [4], Cormode et. al. define a general thresholded problem $(k, f, \tau, \varepsilon)$. A dataset $D$ arrives as a distributed stream across $k$ sites. At any given point in time, the coordinator should be able to determine whether $f(D) \geq \tau$ or $f(D) \leq(1-\varepsilon) \tau$.

In continuous tracking problems, there is no single threshold, and so $f(n)$ is tracked to within an additive $\varepsilon \tau(n)$, where $\tau(n)$ also changes with the dataset $D(n)$. Since $\tau$ is now a function, it needs to be defined; the usual choice is $f$ itself, except for tracking item frequencies and order statistics, for which (following the standard streaming model) $\tau$ is chosen to be $|D|$. That is, the continuous monitoring problem $(k, f, \varepsilon)$ is, at all times $n$ maintain at the coordinator an estimate $\hat{f}(n)$ of $f(n)$ so that $|f(n)-\hat{f}(n)| \leq \varepsilon f(n)$.

To define variability we use ideas implicit in the bounds of Tao et. al. to generalize the $\sum_{t=1}^{n} 1 /|D(t)|$ term in those bounds to apply to arbitrary functions $f(n)$ of the dataset $D(n)$. The intuition behind our definition is as follows. We expect that we would need to communicate each time that $f$ changes by $\pm \varepsilon f$. Further, at each timestep $t$ we know that $f$ changes by $f^{\prime}(t)$. Therefore, at each timestep we should have $\left|\frac{f^{\prime}(t)}{\varepsilon f(t)}\right|$ communication. In sum, we would expect the total number of messages to look like $\sum_{t=1}^{n}\left|\frac{f^{\prime}(t)}{\varepsilon f(t)}\right|$. In sections 3 and 4 we find that, modulo the number $k$ of sites and constant factors, this is indeed the case.

Being a parameter of the problem rather than the stream, we can move the $1 / \varepsilon$ factor out of our definition of variability and bring it back in along with the appropriate functions of $k$ when we state upper and lower bounds for our problem. This permits us to treat the stream parameter $v$ independently of the problem. We also need to handle the case $f=0$ specially, which we can do by communicating at each timestep that case occurs.

With all this in mind, we define the $f$-variability of a stream to be $v(n)=\sum_{t=1}^{n} \min \left\{1,\left|\frac{f^{\prime}(t)}{f(t)}\right|\right\}$. We also write $v^{\prime}(t)=\min \left\{1,\left|\frac{f^{\prime}(t)}{f(t)}\right|\right\}$ to be the increase in variability at time $t$. We say "variability" for $f$-variability in the remainder of this paper.

From a practical perspective, we believe low variability streams to be common. In many database applications the database is interesting primarily because it tends to grow more than it shrinks, so it is common for the size of the dataset to have low variability; as more items are inserted, the rate of change of $|D|$ shrinks relative to itself, and about as many deletions as insertions would be required to keep the ratio constant. In the following subsection, we prove that monotone and nearly monotone functions have low variability and that random walks have low variability in expectation, lending evidence to our belief.

From a theoretical perspective, variability is a way to analyze algorithms for $\varepsilon$ relative error in the face of nonmonotonicity and generate provable worst-case bounds that degrade gracefully as our assumptions about the input become increasingly pessimistic. For our counting problem, it allows us to adapt the existing distributed counting algorithms of Cormode et. al. [4, 5] and Huang et. al. [8] with 
only minor modifications, and the resulting analyses show that the dependence on $k$ and $\varepsilon$ remains unchanged.

\subsection{Interesting cases with small variability}

We start with functions that are nearly monotone in the sense that they are eventually mostly nondecreasing. We make this precise in the theorem statement. Of course, since the $(-f)$-variability of a stream is equal to its $f$-variability, the result holds for functions that are eventually mostly nonincreasing as well.

Theorem 2.1. Let $f^{-}(n)=\sum_{t: f^{\prime}(t)<0}^{n}\left|f^{\prime}(t)\right|$ be the total of negative updates and $f^{+}(n)=\sum_{t: f^{\prime}(t)>0}^{n} f^{\prime}(t)$ be the total of positive updates, so that $f(n)=f^{+}(n)-f^{-}(n)$.

If there is a monotone nondecreasing function $\beta(t) \geq 1$ and a constant $t_{0}$ such that for all $n \geq t_{0}$ we have that $f^{-}(n) \leq \beta(n) f(n)$, then the variability $\sum_{t=1}^{n}\left|f^{\prime}(t) / f(t)\right|$ of the function is $O(\beta(n) \log (\beta(n) f(n)))$.

The proof, which we defer to appendix A, partitions time into intervals over which $f^{+}(t)$ doubles and shows the variability in each interval to be $O(\beta(n))$. When $f(n)$ is strictly monotone, $\beta(n)=1$ suffices, and the theorem reduces to the result claimed in the abstract. As we will see in section 3 , our upper bounds will simplify in the monotone case to those of Cormode et. al. [4, 5] and Huang et. al. [8].

Next, we compute the variability for two random input classes considered by Liu et. al. [10,11]. This will permit us to decouple the randomness of their algorithms from the randomness of their inputs. This means, for example, that even our deterministic algorithm of section 3 has $o(n)$ cost in expectation for these input classes. The first random input class we consider is the symmetric random walk.

THEOREM 2.2. If $f^{\prime}(t)$ is a stream of i.i.d. \pm 1 coin flips then the expected variability $E(v(n))=O(\sqrt{n} \log n)$.

Proof. The update sequence defines a random walk for $f(t)$, and the expected variability is

$$
\sum_{t=1}^{n} P(f(t)=0)+\sum_{t=1}^{n} \sum_{s=1}^{t} 2 P(f(t)=s) / s
$$

We use the following fact, mentioned and justified in Liu et. al. [10]:

FACT 2.3. For any $t \geq 1$ and $s \in[-t, t]$ we have that $P(f(t)=s) \leq c_{1} / \sqrt{t}$, where $c_{1}$ is some constant.

Together, these show the expected cost to be at most

$c_{1} \sum_{t=1}^{n}\left(1+2 H_{t}\right) / \sqrt{t} \leq c_{2} \log (n) \sum_{t=1}^{n} 1 / \sqrt{t} \leq c_{3} \log (n) \sqrt{n}$

since $\left(1+2 H_{n}\right) \leq \frac{c_{2}}{c_{1}} \log (n)$ and $\sum_{t=1}^{n} \frac{1}{\sqrt{t}} \leq \frac{c_{3}}{2 c_{2}} \int_{1}^{n} \frac{1}{\sqrt{t}} d t$.

The second random input class we consider is i.i.d. increments with a common drift rate of $\mu>0$. The case $\mu<0$ is symmetric. We assume that $\mu$ is constant with respect to $n$. The proof is a simple application of Chernoff bounds and is deferred to appendix B.

THEOREM 2.4. If $f^{\prime}(t)$ is a sequence of i.i.d. \pm 1 random variables with $P\left(f^{\prime}(t)=1\right)=(1+\mu) / 2$ then the expected variability $E(v(n))=O\left(\frac{\log n}{\mu}\right)$.

\section{Remarks.}

We can restate the results of Liu et. al. [10,11] and Tao et. al. [13] in terms of variability. For unbiased coin flips, Liu et. al. obtain an algorithm that uses $O\left(\frac{\sqrt{k}}{\varepsilon} \sqrt{n} \log n\right)$ messages (of size $O(\log n)$ bits each) in expectation, and for biased coin flips with constant $\mu$, an algorithm that uses $O\left(\frac{\sqrt{k}}{\varepsilon} \frac{1}{|\mu|}(\log n)^{1+c}\right)$ messages in expectation. If we rewrite these bounds in terms of expected variability, they become $O\left(\frac{\sqrt{k}}{\varepsilon} E(v(n))\right)$ and $O\left(\frac{\sqrt{k}}{\varepsilon}(\log n)^{c} E(v(n))\right)$, respectively. In the next section, we obtain (when $k=O\left(1 / \varepsilon^{2}\right)$ ) a randomized bound of $O\left(\frac{\sqrt{k}}{\varepsilon} v(n)\right)$. In marked contrast to the bounds of Liu et. al., our bound is a worst-case lower bound that is a function of $v(n)$; if the input happens to be generated by fair coin flips, then our expected cost happens to be $O\left(\frac{\sqrt{k}}{\varepsilon} \sqrt{n} \log n\right)$.

The results of Tao et. al. are for a different setting, but having used them as a starting point for our definition of variability we can restate their bounds terms of the $|D|$-variability $v(n)$ : for the problem of tracking the historical record of order statistics, they obtain a lower bound of $\Omega\left(\frac{1}{\varepsilon} v(n)\right)$, an offline upper bound of $O\left(\left(\frac{1}{\varepsilon} \log ^{2} \frac{1}{\varepsilon}\right) v(n)\right)$, and an online upper bound of $O\left(\frac{1}{\varepsilon^{2}} v(n)\right)$. We adapt ideas from both their upper and lower bounds in sections 3 and 4 .

\section{UPPER BOUNDS}

In this section we develop deterministic and randomized algorithms for maintaining at the coordinator an estimate $\hat{f}(n)$ for $f(n)$ that is usually good. In particular, for deterministic algorithms we require the deterministic guarantee that $\forall n,|f(n)-\hat{f}(n)| \leq \varepsilon f(n)$, and for randomized algorithms we require the probabilistic guarantee that $\forall n$, $P(|f(n)-\hat{f}(n)| \leq \varepsilon f(n)) \geq 2 / 3$. We obtain deterministic and randomized upper bounds of $O\left(\frac{k}{\varepsilon} v(n)\right)$ and $O\left(\left(k+\frac{\sqrt{k}}{\varepsilon}\right) v(n)\right)$ messages, respectively. For comparison, the analogous algorithms of Cormode et. al. [4, 5] and Huang et. al. [8] for the monotone case use $O\left(\frac{k}{\varepsilon} \log n\right)$ and $O\left(\left(k+\frac{\sqrt{k}}{\varepsilon}\right) \log n\right)$ messages, respectively.

For our upper bounds we assume that $f^{\prime}(n)$ is always \pm 1 . If $\left|f^{\prime}(n)\right|>1$ we could simulate it with $\left|f^{\prime}(n)\right|$ arrivals of \pm 1 updates with $O\left(\log \max f^{\prime}(n)\right)$ overhead, as shown in appendix C.

\subsection{Partitioning time}

We use an idea from Tao et. al. [13] to first divide time into manageable blocks. At the end of each block we know the values $n$ and $f(n)$ exactly. Within each block, we know these values only approximately. The division into blocks is deterministic and the same for both our deterministic and randomized algorithms. Our division ensures that the change in $v(n)$ over each block is at least $1 / 5$, which simplifies our analysis. Specifically, we prove

THEOREM 3.1. There is an algorithm to divide time into blocks $B_{0}, B_{1}, \ldots$, where $B_{j}=\left[n_{j}+1, n_{j+1}\right]$, such that $n_{0}=0$, the change $v_{j}=v\left(n_{j+1}\right)-v\left(n_{j}\right)$ in variability over each block $B_{j}$ is at least $1 / 5$, the value $f(n)$ within block $B_{j}$ differs from its start value $f\left(n_{j}\right)$ by no more than $\max \left\{\frac{5}{2}\left|f\left(n_{j}\right)\right|, k\right\}$, and the partitioning algorithm uses at most $25 k v+3 k$ messages of size $O(\log n)$ bits each.

The algorithm is as follows. A precise pseudocode description appears in appendix D. 
- The coordinator requests the sites' values $c_{i}$ and $f_{i}$ at times $n_{0}=0, n_{1}, n_{2}, \ldots$ and then broadcasts a value $r$. These values will be defined momentarily.

- Each site $i$ maintains a variable $c_{i}$ that counts the number of stream updates $f^{\prime}(n)$ it received since the last time it sent $c_{i}$ to the coordinator. It also maintains $f_{i}$ that counts the change in $f$ it received since the last broadcast $n_{j}$. Whenever $c_{i}=\left\lceil 2^{r-1}\right\rceil$, site $i$ sends $c_{i}$ to the coordinator. This is in addition to replying to requests from the coordinator.

- The coordinator maintains a variable $\hat{t}$. After broadcasting $r, \hat{t}$ is reset to zero. Whenever site $i$ sends $c_{i}$, the coordinator updates $\hat{t}=\hat{t}+c_{i}$ and $n_{j}=n_{j}+c_{i}$.

- The coordinator also maintains variables $\hat{f}, j$, and $t_{j}$. At the first time $n_{j}>n_{j-1}$ at which $\hat{t} \geq t_{j}$, the coordinator requests the $c_{i}$ and $f_{i}$ values, updates $\hat{f}$ and $r$, sets $t_{j+1}=\left\lceil 2^{r-1}\right\rceil k$, broadcasts $r$, and increments $j$.

- When $r$ is updated at the end of time $n_{j}$, it is set to $r$ if $2^{r} 2 k \leq\left|f\left(n_{j}\right)\right|<2^{r} 4 k$ and zero if $\left|f\left(n_{j}\right)\right|<4 k$.

Proof. Algebra tells us some facts:

- $\left\lceil 2^{r-1}\right\rceil k \leq n_{j+1}-n_{j} \leq 2^{r} k$.

- $\left|f(n)-f\left(n_{j}\right)\right| \leq 2^{r} 5 k$ for all $n$ in $B_{j}$.

- If $r \geq 1$ then $\left|f(n)-f\left(n_{j}\right)\right| \geq 2^{r} k$ for all $n$ in $B_{j}$.

The total number of messages sent in block $B_{j}$ is at most $5 k$ : we have at most $2 k$ updates from sites, $k$ requests from the coordinator, $k$ replies from each site, and $k$ broadcast at $n_{j+1}$.

The change in variability $v_{j}$ over block $B_{j}$ is

$$
v_{j}=\sum_{t=n_{j}+1}^{n_{j+1}} \frac{1}{\min \{1,|f(t)|\}} \geq 2^{r} k / 2^{r} 5 k \geq 1 / 5
$$

And therefore the total number of messages is bounded by $25 k v+3 k$.

\subsection{Estimation inside blocks}

What remains is to estimate $f(n)$ within a given block, which we do by adapting the existing monotone algorithms of Cormode et. al. [4, 5] and Huang et. al. [8] to positive and negative updates within a block. Both our deterministic and randomized algorithms use the following template, changing only CONDITION, MESSAGE, and UPDATE:

- Site $i$ maintains a variable $d_{i}$ that tracks the drift at site $i$, defined as the sum of $f^{\prime}(n)$ updates received at site $i$ during the block. That is, $f(n)-f\left(n_{j}\right)=\sum_{i} d_{i}$.

- Site $i$ also maintains a variable $\delta_{i}$ that tracks the change in $d_{i}$ since the last time site $i$ sent a MESSAGE. $\delta_{i}$ is initially zero.

- The coordinator maintains an estimate $\hat{d}_{i}$ for each value $d_{i}$. These are initially zero. It also defines two estimates based on these $\hat{d}_{i}$ :

- For the global drift: $\hat{d}=\sum_{i} \hat{d}_{i}$.

- For $f(n): \hat{f}(n)=f\left(n_{j}\right)+\hat{d}(n)$.

- When site $i$ receives stream update $f^{\prime}(n)$, it updates $d_{i}$. It then checks its CONDITION. If true, it sends a MESSAGE to the coordinator and resets $\delta_{i}=0$.

- When the coordinator receives a MESSAGE from a site $i$ it UPDATEs its estimates.

\subsection{The deterministic algorithm}

Our method guarantees that $|f(n)-\hat{f}(n)| \leq \varepsilon|f(n)|$ holds at all times $n$. It uses $O(k v / \varepsilon)$ messages in total. In the following, $r$ is the value of $r$ for the current block.

- Condition: true if $\left|\delta_{i}\right|=1$ and $r=0$, or if $\left|\delta_{i}\right| \geq \varepsilon 2^{r}$. Otherwise, false.

- Message: the new value of $d_{i}$.

- UPDATE: set $\hat{d}_{i}=d_{i}$.

Proof. Let $\delta=\sum_{i} \delta_{i}$ be the error with which $\hat{d}$ estimates $d=\sum_{i} d_{i}$. The error in $\hat{f}$ is

$$
\begin{aligned}
|f(n)-\hat{f}(n)| & =\left|\left(f\left(n_{j}\right)+d(n)\right)-\left(\hat{f}\left(n_{j}\right)+d(n)+\delta(n)\right)\right| \\
& =|\delta(n)|
\end{aligned}
$$

When $r \geq 1$ we have $\left|B_{j}\right| \leq 2^{r} k$, and we always have that $\delta \leq\left|B_{j}\right|$. Since we constrain $\delta_{i}<\varepsilon 2^{r}$ at the end of each timestep, we have $|f(n)-\hat{f}(n)|<\varepsilon 2^{r} k \leq \varepsilon|f(n)|$.

We also use at most $2 k / \varepsilon$ messages for the block. If $r=0$ then the number of messages is at most $k$. If $r \geq 1$, then since a site must receive $\varepsilon 2^{r}$ new stream updates to send a new message, and since there are at most $2^{r} k$ stream updates in the block, there are at most $k / \varepsilon$ messages.

In each block the change in $v$ is at least $1 / 5$, so the total number of messages is at most $5 k v / \varepsilon$.

\subsection{The randomized algorithm}

Our method uses $O(\sqrt{k} v / \varepsilon)$ messages (plus the time partitioning) and guarantees $P(|f(n)-\hat{f}(n)|>\varepsilon|f(n)|)<1 / 3$ holds for all times $n$.

The idea is to estimate the sums $d_{i}^{+}$and $d_{i}^{-}$of +1 and -1 updates separately. The estimators for those values are independent and monotone, so we can use the method of Huang et. al. [8] to estimate the two and then combine them.

Specifically, the coordinator and each site run two independent copies $A^{+}$and $A^{-}$of the algorithm. Whenever $f^{\prime}(n)=+1$ arrives at site $i, \mathrm{a}+1$ is fed into algorithm $A^{+}$ at site $i$. Whenever $f^{\prime}(n)=-1$ arrives at site $i$, a +1 is fed into algorithm $A^{-}$at site $i$. So the drifts $d_{i}^{+}$and $d_{i}^{-}$ at every site will always be nonnegative. At the coordinator, the estimates $\hat{d}_{i}^{ \pm}$and $\hat{d}^{ \pm}$are tracked independently also. However, the coordinator also defines $\hat{d}=\hat{d}^{+}-\hat{d}^{-}$and $\hat{f}(n)=f\left(n_{j}\right)+\hat{d}(n)$. The definitions for algorithm $A^{ \pm}$are

- Condition: true with probability $p=\min \left\{1, \frac{3}{\varepsilon 2^{r} k^{1 / 2}}\right\}$.

- Message: the new value of $d_{i}^{ \pm}$.

- UpDATE: set $\hat{d}_{i}^{ \pm}=d_{i}^{ \pm}-1+1 / p$.

Proof. The following fact 3.2 is lemma 2.1 of Huang et. al. [8]. For each block $B_{j}$ our algorithm effectively divides the stream $f^{\prime}\left(B_{j}\right)$ into two streams $\left|f^{\prime}\left(B_{j}^{ \pm}\right)\right|$. Since these streams consist of +1 increments only we run the algorithm of Huang et. al. separately on each of them. At any time $n$, stream $\left|f^{\prime}\left(B_{j}^{ \pm}\right)\right|$has seen $d_{i}^{ \pm}(n)$ increments at site $i$, and lemma 2.1 of Huang et. al. guarantees that the estimates $\hat{d}_{i}^{ \pm}(n)$ for the counts $d_{i}^{ \pm}(n)$ are good.

FACT 3.2. $E\left(\hat{d}_{i}^{ \pm}\right)=d_{i}^{ \pm}$and $\operatorname{Var}\left(\hat{d}_{i}^{ \pm}\right) \leq 1 / p^{2}$.

This means that $E\left(\hat{d}^{ \pm}\right)=\sum_{i} E\left(\hat{d}_{i}^{ \pm}\right)=\sum_{i} d_{i}^{ \pm}$, and therefore that $E(\hat{d})=\sum_{i} E\left(d_{i}^{+}-d_{i}^{-}\right)=\sum_{i} d_{i}$. Since the estimators 
$\hat{d}_{i}^{ \pm}$are independent, the variance of the global drift is at most $2 k / p^{2}$. By Chebyshev's inequality,

$$
P\left(|\delta(n)|>\varepsilon 2^{r} k\right) \leq \frac{2 k / p^{2}}{\left(\varepsilon 2^{r} k\right)^{2}}<1 / 3
$$

Further, the expected cost of block $B_{j}$ is bounded above by $p\left|B_{j}\right| \leq\left(3 / \varepsilon 2^{r} k^{1 / 2}\right)\left(2^{r} 2 k\right) \leq 30 k^{1 / 2} v_{j} / \varepsilon$.

\section{LOWER BOUNDS}

In this section we show that the dependence on $v$ is essentially necessary by developing deterministic and randomized lower bounds on space+communication that hold even when $v$ is small. Admittedly, this is not as pleasing as a pure communication lower bound would be. On the other hand, a distributed monitoring algorithm with high space complexity would be impractical for monitoring sensor data, network traffic patterns, and other applications of the model. Note that in terms of space+communication, our deterministic lower bound is tight up to factors of $k$, and our randomized lower bound is within a factor of $\log (n)$ of that.

For these lower bounds we use a slightly different problem. We call this problem the tracing problem. The streaming model for the tracing problem is the standard turnstile streaming model with updates $f^{\prime}(n)$ arriving online. The problem is to maintain in small space a summary of the sequence $f$ so that, at any current time $n$, if we are given an earlier time $t$ as a query, we can return an estimate $\hat{f}(t)$ so that $P(|f(t)-\hat{f}(t)| \leq \varepsilon f(t))$ is large (one in the deterministic case, $2 / 3$ in the randomized case). We call this the tracing problem because our summary traces $f$ through time, so that we can look up earlier values.

In appendix $\mathrm{E}$ we show that a space lower bound for the tracing problem implies a space+communication lower bound for the distributed tracking problem. Here, we develop deterministic and randomized space lower bounds for the tracing problem.

\subsection{The deterministic bound}

The deterministic lower bound that follows is similar in spirit to the lower bound of Tao et. al. [13]. It uses a simple information-theoretic argument.

ThEOREM 4.1. Let $\varepsilon=1 / m$ for some integer $m \geq 2$, let $n \geq 2 m$, let $c<1$ constant, and let $r \leq n^{c}$ and even. If a deterministic summary $S(f)$ guarantees for all $t \leq n$ that $|f(t)-\hat{f}(t)| \leq \varepsilon f(t)$, even only for sequences for which $v(n)=\frac{6 m+9}{2 m+6} \varepsilon r$, then that summary must use $\Omega\left(\frac{\log n}{\varepsilon} v(n)\right)$ bits of space.

The full proof appears in appendix F. At a high level, the sequences in the family take only values $m$ or $m+3$, and each sequence is defined by $r$ of the $n$ timesteps. If the new timestep $t$ is one of the $r$ chosen for our sequence, then we flip from $m$ to $m+3$ or vice-versa. All of these sequences are unique and there are $2^{\Omega(r \log n)}$ of them.

\subsection{The randomized bound}

We use a construction similar to the one in our deterministic lower bound to produce a randomized lower bound. In order to make the analysis simple we forego a single variability value for all sequences in our constructed family, but still maintain that they all have low variability. $C$ is a universal constant to be defined later.
Theorem 4.2. Choose $\varepsilon \leq 1 / 2, v \geq 32400 \varepsilon \ln C$, and $n>3 v / \varepsilon$. If a summary $S(f)$ guarantees for all $t \leq n$ that $P(|f(t)-\hat{f}(t)| \leq \varepsilon f(t)) \geq 99 / 100$, even only for sequences for which $v(n) \leq v$, then that summary must use $\Omega(v / \varepsilon)$ bits of space.

We prove this theorem in two lemmas. In the first lemma, we reduce the claim to a claim about the existence of a hard family of sequences. In the second lemma we show the existence of such a family.

First a couple of definitions. For any two sequences $f$ and $g$ define the number of overlaps between $f$ and $g$ to be the number of positions $1 \leq t \leq n$ for which

$$
|f(t)-g(t)| \leq \varepsilon \max \{f(t), g(t)\}
$$

Say that $f$ and $g$ match if they have at least $\frac{6}{10} n$ overlaps.

LEMMA 4.3. Let $\mathcal{F}$ be a family of sequences of length $n$ and variabilities $\leq v$ such that no two sequences in $\mathcal{F}$ match. If a summary $S(f)$ guarantees for all $f$ in $\mathcal{F}$ and all $t \leq n$ that $P(|f(t)-\hat{f}(t)| \leq \varepsilon f(t)) \geq 99 / 100$, then that summary must use $\Omega(\log |\mathcal{F}|)$ bits of space.

The full proof appears in appendix G. At a high level, if $S(f)$ is the summary for a sequence $f$, we can use it to generate an approximation $\hat{f}$ that at least $90 \%$ of the time overlaps with $f$ in at least $\frac{9}{10} n$ positions. Since no two sequences in $\mathcal{F}$ overlap in more than $\frac{6}{10} n$ positions, at least $90 \%$ of the time we can determine $f$ given $\hat{f}$. We then solve the one-way Index $N$ problem by deterministically generating $\mathcal{F}$ and sending a summary $S(f(x))$, where $x$ is Alice's input of size $N=\log _{2}|\mathcal{F}|$, and $f(x)$ is the $x$ th sequence in $\mathcal{F}$.

Lemma 4.4. For all $\varepsilon \leq 1 / 2$, all $v \geq 32400 \varepsilon \ln C$, and all $n>3 v / \varepsilon$, there is a family $\mathcal{F}$ of size $e^{\Omega(v / \varepsilon)}$ of sequences of size $n$ such that no two sequences match and every sequence has variability at most $v$.

The full proof appears in appendix H. At a high level, sequences again switch between $m=1 / \varepsilon$ and $m+3$, except that these switches are chosen independently. We model the overlap with a Markov chain; the overlap between any two sequences is the sum over times $t$ of a function $y$ applied to the states of a chain modeling their interaction. We then apply a result of Chung, Lam, Liu, and Mitzenmacher [2] to show that the probability that any two sequences match is low. Lastly, we show that not too many sequences have variability more than $v$, by proving that they usually don't switch between $m$ and $m+3$ many times.

\section{VARIABILITY AS A FRAMEWORK}

In section 2 we proposed $f$-variability as a way to analyze algorithms for the continuous monitoring problem $(k, f, \varepsilon)$ over general update streams. However, our discussion so far has focused on distributed counting. In this final section we revisit the suitability of our definition by mentioning extensions to tracking other functions of a dataset defined by a distributed update stream. We include fuller discussions of these extensions in the appendices.

\subsection{Tracking item frequencies}

We can extend our deterministic algorithm of section 3 to the problem of tracking item frequencies, in a manner similar 
to that in which Yi and Zhang $[16,17]$ extend the ideas of Cormode et. al. [4] to this problem. The definition of this problem, the required changes to our algorithm of section 3 needed to solve it, and a discussion of the difficulties in finding a randomized algorithm, are discussed in appendix I.

\subsection{Aggregate functions with one site}

In this subsection we consider general single-integer-valued functions $f$ of a dataset, including highly non-linear functions such as high-order frequency moments. When there is a single site, the site always knows the exact value of $f(n)$, and the only issue is updating the coordinator to have an approximation $\hat{f}(n)$ so that $|f(n)-\hat{f}(n)| \leq \varepsilon f(n)$ for all $n$. We can show that this problem of tracking $f$ to $\varepsilon$ relative error when $k=1$ has an $O\left(\frac{1}{\varepsilon} v(n)\right)$-word upper bound, where here $v(n)$ is the $f$-variability. The algorithm is: whenever $|f-\hat{f}|>\varepsilon f$, send $f$ to the coordinator. The proof is a simple potential argument and is deferred to appendix J. Along with our lower bounds of section 4, this upper bound lends evidence to our claim that variability captures some or all of the difficulty of communicating changes in general functions $f$ that are due to the non-monotonicity of the input stream.

\section{Acknowledgments}

We thank the anonymous reviewers for their many useful comments and suggestions.

Research supported in part by NSF grants CCF-0916574; IIS-1065276; CCF-1016540; CNS-1118126; CNS-1136174; US-Israel BSF grant 2008411, OKAWA Foundation Research Award, IBM Faculty Research Award, Xerox Faculty Research Award, B. John Garrick Foundation Award, Teradata Research Award, and Lockheed-Martin Corporation Research Award. This material is also based upon work supported by the Defense Advanced Research Projects Agency through the U.S. Office of Naval Research under Contract N00014-11-1-0392. The views expressed are those of the author and do not reflect the official policy or position of the Department of Defense or the U.S. Government.

\section{REFERENCES}

[1] C. Arackaparambil, J. Brody, and A. Chakrabarti. Functional monitoring without monotonicity. In Automata, Languages and Programming, pages 95-106. Springer, 2009.

[2] K.-M. Chung, H. Lam, Z. Liu, and M. Mitzenmacher. Chernoff-hoeffding bounds for markov chains: Generalized and simplified. arXiv preprint arXiv:1201.0559, 2012.

[3] G. Cormode and S. Muthukrishnan. An improved data stream summary: the count-min sketch and its applications. Journal of Algorithms, 55(1):58 - 75, 2005.

[4] G. Cormode, S. Muthukrishnan, and K. Yi. Algorithms for distributed functional monitoring. In Proceedings of the Nineteenth Annual ACM-SIAM Symposium on Discrete Algorithms, SODA '08, pages 1076-1085, Philadelphia, PA, USA, 2008. Society for Industrial and Applied Mathematics.

[5] G. Cormode, S. Muthukrishnan, and K. Yi. Algorithms for distributed functional monitoring. ACM Transactions on Algorithms (TALG), 7(2):21, 2011.
[6] S. Ganguly and A. Majumder. Cr-precis: A deterministic summary structure for update data streams. CoRR, abs/cs/0609032, 2006.

[7] S. Ganguly and A. Majumder. Cr-precis: A deterministic summary structure for update data streams. In B. Chen, M. Paterson, and G. Zhang, editors, Combinatorics, Algorithms, Probabilistic and Experimental Methodologies, volume 4614 of Lecture Notes in Computer Science, pages 48-59. Springer Berlin Heidelberg, 2007.

[8] Z. Huang, K. Yi, and Q. Zhang. Randomized algorithms for tracking distributed count, frequencies, and ranks. In Proceedings of the 31st symposium on Principles of Database Systems, pages 295-306. ACM, 2012.

[9] E. Kushilevitz and N. Nisan. Communication Complexity. Cambridge University Press, New York, NY, USA, 1997.

[10] Z. Liu, B. Radunović, and M. Vojnović. Continuous distributed counting for non-monotonic streams. In Technical Report MSR-TR-2011-128, 2011.

[11] Z. Liu, B. Radunović, and M. Vojnović. Continuous distributed counting for non-monotonic streams. In Proceedings of the 31st symposium on Principles of Database Systems, pages 307-318. ACM, 2012.

[12] S. Muthukrishnan. Data streams: Algorithms and applications. Now Publishers Inc, 2005.

[13] Y. Tao, K. Yi, C. Sheng, J. Pei, and F. Li. Logging every footstep: quantile summaries for the entire history. In Proceedings of the 2010 ACM SIGMOD International Conference on Management of data, SIGMOD '10, pages 639-650, New York, NY, USA, 2010. ACM.

[14] D. P. Woodruff and Q. Zhang. Tight bounds for distributed functional monitoring. CoRR, abs/1112.5153, 2011.

[15] D. P. Woodruff and Q. Zhang. Tight bounds for distributed functional monitoring. In Proceedings of the 44th symposium on Theory of Computing, pages 941-960. ACM, 2012.

[16] K. Yi and Q. Zhang. Optimal tracking of distributed heavy hitters and quantiles. In Proceedings of the Twenty-eighth ACM SIGMOD-SIGACT-SIGART Symposium on Principles of Database Systems, PODS '09, pages 167-174, New York, NY, USA, 2009. ACM.

[17] K. Yi and Q. Zhang. Optimal tracking of distributed heavy hitters and quantiles. Algorithmica, 65(1):206-223, 2013.

\section{APPENDIX}

\section{A. VARIABILITY OF NEARLY MONOTONE FUNCTIONS, THEOREM 2.1}

\footnotetext{
ThEOREM A.1. Let $f^{-}(n)=\sum_{t: f^{\prime}(t)<0}^{n}\left|f^{\prime}(t)\right|$ be the total of negative updates and $f^{+}(n)=\sum_{t: f^{\prime}(t)>0}^{n} f^{\prime}(t)$ be the total of positive updates, so that $f(n)=f^{+}(n)-f^{-}(n)$.

If there is a monotone nondecreasing function $\beta(t) \geq 1$ and a constant $t_{0}$ such that for all $n \geq t_{0}$ we have that $f^{-}(n) \leq \beta(n) f(n)$, then the variability $\sum_{t=1}^{n}\left|f^{\prime}(t) / f(t)\right|$ of the function is $O(\beta(n) \log (\beta(n) f(n)))$.
} 
Proof. Note that $f^{-}(n) \leq \beta(n) f(n)$ and $\beta(n) \geq 1$ imply that $f(n) \geq 0$. For $i=1, \ldots, k$, define $t_{i}$ to be the earliest time $t$ such that $f^{+}\left(t_{i}\right)>2 f^{+}\left(t_{i-1}\right)$, where $k$ is the smallest index such that $t_{k}>n$. (If $k$ is undefined, define $k=n+1$.)

The cost $\sum_{t=1}^{t_{0}-1}\left|f^{\prime}(t) / f(t)\right|$ is constant. We bound the cost $\sum_{t=t_{0}}^{n}\left|f^{\prime}(t)\right| / f(t)$ as follows. We partition the interval $\left[t_{0}, t_{k}\right)$ into subintervals $\left[t_{0}, t_{1}\right), \ldots,\left[t_{k-1}, t_{k}\right)$ and sum over the times $t$ in each one. There are at most $1+\log f^{+}(n)$ of these subintervals.

$$
\begin{aligned}
\sum_{t=t_{0}}^{n} \frac{\left|f^{\prime}(t)\right|}{f(t)} & \leq \sum_{i=1}^{k} \sum_{t=t_{i-1}}^{t_{i}-1} \frac{\left|f^{\prime}(t)\right|}{f(t)} \\
& \leq \sum_{i=1}^{k} \frac{1+\beta(n)}{f^{+}\left(t_{i-1}\right)} \sum_{t=t_{i-1}}^{t_{i}-1}\left|f^{\prime}(t)\right| \\
& \leq \sum_{i=1}^{k}(1+\beta(n)) \frac{f^{+}\left(t_{i}-1\right)+f^{-}\left(t_{i}-1\right)}{f^{+}\left(t_{i-1}\right)} \\
& \leq 4(1+\beta(n))\left(1+\log f^{+}\left(t_{i}-1\right)\right) \\
& \leq 4(1+\beta(n))(1+\log (2(1+\beta(n)) f(n)))
\end{aligned}
$$

where the second inequality follows from

$$
f^{+}(t)=f(t)+f^{-}(t) \leq(1+\beta(n)) f(t)
$$

and the fourth holds because

$$
f^{+}\left(t_{i}-1\right)+f^{-}\left(t_{i}-1\right) \leq 2 f^{+}\left(t_{i}-1\right)
$$

(because $f(t) \geq 0$ implies $f^{+}(t) \geq f^{-}(t)$ ).

\section{B. VARIABILITY OF BIASED COIN FLIPS, THEOREM 2.4}

TheOREM B.1. If $f^{\prime}(t)$ is a sequence of i.i.d. \pm 1 random variables with $P\left(f^{\prime}(t)=1\right)=(1+\mu) / 2$ then the expected variability $E(v(n))=O\left(\frac{\log n}{\mu}\right)$.

Proof. We show that, with high probability, $f(t) \geq \mu t / 2$ for times $t \geq t_{0}=t_{0}(n)$ when $n$ is large enough with respect to $\mu$.

We write $f(t)=-t+2 Y_{t}$, where $Y_{t}=\sum_{s=1}^{t} y_{s}$, and $y_{s}$ is a Bernoulli variable with mean $\frac{1+\mu}{2}$. We have that $P(f(t) \leq \mu t / 2)=P\left(Y_{t} \leq \frac{2+\mu}{4} t\right)$ and that $E\left(Y_{t}\right)=\frac{1+\mu}{2} t$. Further, $P\left(Y_{t} \leq \frac{2+\mu}{4} t\right) \leq \exp (-\mu t / 16)$ using a Chernoff bound. Let $A$ be the event $\exists t \geq t_{0}(f(t) \leq \mu t / 2)$. Then $P(A) \leq \sum_{t=t_{0}}^{n} e^{-\mu t / 16}$ by the union bound. We can upper bound this sum by

$$
\sum_{t=t_{0}}^{n} e^{-\mu t / 16} \leq e^{-\mu t_{0} / 16}+\int_{t_{0}}^{n} e^{-\mu t / 16} d t \leq 17 e^{-\mu t_{0} / 16} / \mu
$$

Taking $t_{0}=(16 / \mu) \ln (17 n / \mu)$ gives us $P(A) \leq 1 / n$. Thus

$$
\begin{aligned}
& E\left(\sum_{t=1}^{n} \min \left\{1,\left|\frac{f^{\prime}(t)}{f(t)}\right|\right\}\right) \\
\leq & t_{0}+\left(\frac{1}{n}\right) n+\left(1-\frac{1}{n}\right) \sum_{t=t_{0}}^{n} \frac{2}{\mu t}
\end{aligned}
$$

$=O\left(\frac{\log n}{\mu}\right)$, yielding the theorem.

\section{SIMULATING LARGE UPDATES, SECTION 3}

We noted in section 3 that we can simulate $\left|f^{\prime}(n)\right|>1$ with $\left|f^{\prime}(n)\right|$ arrivals of \pm 1 updates with $O\left(\log \max f^{\prime}(n)\right)$ overhead. To simplify notation we define $1 / f(n)=1$ when $f(n)=0$ and assume that $f(n) \geq 0$ always.

TheOREM C.1. $\sum_{t=1}^{f^{\prime}(n)} \frac{1}{f(n-1)+t} \leq \frac{f^{\prime}(n)}{f(n)} H\left(f^{\prime}(n)\right)$ when $f^{\prime}(n)>1$ and $\sum_{t=0}^{-1-f^{\prime}(n)} \frac{1}{f(n)+t} \leq \frac{\left|f^{\prime}(n)\right|}{f(n)}$ when $f^{\prime}(n)<-1$, where $H(x)$ is the $x$ th harmonic number.

Proof. If $f^{\prime}(n)>1$, then

$$
\begin{aligned}
\sum_{t=1}^{f^{\prime}(n)} \frac{1}{f(n-1)+t} & =\frac{1}{f(n)} \sum_{t=1}^{f^{\prime}(n)} \frac{f(n-1)+f^{\prime}(n)}{f(n-1)+t} \\
& \leq \frac{1}{f(n)} \sum_{t=1}^{f^{\prime}(n)} \frac{f^{\prime}(n)}{t}
\end{aligned}
$$

If $f^{\prime}(n)<-1$ and $f(n) \geq 1$, then

$$
\sum_{t=0}^{-1-f^{\prime}(n)} \frac{1}{f(n)+t} \leq \sum_{t=0}^{-1-f^{\prime}(n)} \frac{1}{f(n)}=\frac{\left|f^{\prime}(n)\right|}{f(n)}
$$

and if $f(n)=0$ we have $\frac{\left|f^{\prime}(n)\right|}{f(n)}=\left|f^{\prime}(n)\right| \cdot(1 / f(n))$.

\section{BLOCK ALGORITHM, THEOREM 3.1}

The algorithm of theorem 3.1 that divides time into blocks has two unique processes, one that runs at the coordinator and one that runs at each of the $k$ sites. Implicit assumptions of the model are that all of the initialization occurs prior to receiving any stream items, and that no stream items arrive while communication occurs.

The following algorithm, figure 1 , is a precise pseudocode description of the coordinator process.

Initialize $j=0, n_{j}=0, t_{j+1}=k, \hat{t}=0, \hat{f}=0$, and $r=0$.

Request sites' values $c_{i}$ and $f_{i}$, and broadcast $r$.

on receiving $c_{i}$ from site $i$ do

Update $\hat{t}=\hat{t}+c_{i}$ and $n_{j}=n_{j}+c_{i}$.

if $\hat{t} \geq t_{j+1}$ then

Increment $j$.

Request sites' values $c_{i}$ and $f_{i}$.

Update $\hat{f}=\hat{f}+\sum_{i=1}^{k} f_{i}$. (Now, $\left.\hat{f}=f.\right)$

Update $n_{j}=n_{j}+\sum_{i=1}^{k} c_{i}$. (Now, $n_{j}=n$.)

if $\left|f\left(n_{j}\right)\right|<4 k$ then

Update $r=0$.

else

Update $r=\left\lfloor\log _{2}\left(\left|f\left(n_{j}\right)\right| / 2 k\right)\right\rfloor$.

(Now, $2^{r} 2 k \leq\left|f\left(n_{j}\right)\right|<2^{r} 4 k$.)

Set $t_{j+1}=\left\lceil 2^{r-1}\right\rceil k$.

Broadcast $r$.

Figure 1: Coordinator process for algorithm of theorem 3.1.

The following algorithm, figure 2 , is a precise pseudocode description of the process running at site $i$. 
Initialize $c_{i}=0, f_{i}=0$, and $r=0$.

on receiving an update $f^{\prime}(n)$ do

Update $c_{i}=c_{i}+1$ and $f_{i}=f_{i}+f^{\prime}(n)$.

if $c_{i}=\left[2^{r-1}\right\rceil$ then

Send $c_{i}$ to the coordinator and reset $c_{i}=0$.

on receiving a request from the coordinator do

Send the coordinator $c_{i}$ and $f_{i}$.

on receiving a broadcast $r$ from the coordinator do Update $r$ to the broadcasted value.

Reset $c_{i}=0$ and $f_{i}=0$.

Figure 2: Site $i$ process for algorithm of theorem 3.1.

\section{E. REDUCING DISTRIBUTED TRACKING TO TRACING, SECTION 4}

Lemma E.1. Fix some $\varepsilon$. Suppose that the tracing problem has an $\Omega\left(L_{\varepsilon}(n)\right)$-bit space deterministic lower bound. Also suppose that there is a deterministic algorithm $A$ for the distributed tracking problem that uses $C_{\varepsilon}(n)$ bits of communication and $S_{\varepsilon}(n)$ bits of space at the site and coordinator combined. Then we must have $C+S=\Omega(L)$.

Further, if we replace "deterministic" with "randomized" in the preceding paragraph, the claim still holds.

ProOF. Toward a contradiction, suppose that for all constants $c<1$ and all $n_{0}$ there is an $n>n_{0}$ such that $C(n)+S(n)<c L(n)$. Then we can write an algorithm $B$ for the tracing problem that uses $L^{\prime}(n)<c L(n)$ bits of space: simulate $A$, recording all communication, and on a query $t$, play back the communication that occurred through time $t$.

At no point did we use the fact that $A$ guarantees that $P(|f(t)-\hat{f}(t)| \leq \varepsilon f(t))=1$, so the claim still holds if we change the correctness requirement to $P \geq 2 / 3$.

\section{F. DETERMINISTIC LOWER BOUND, THEOREM 4.1}

ThEOREM F.1. Let $\varepsilon=1 / m$ for some integer $m \geq 2$, let $n \geq 2 m$, let $c<1$ constant, and let $r \leq n^{c}$ and even. If a deterministic summary $S(f)$ guarantees for all $t \leq n$ that $|f(t)-\hat{f}(t)| \leq \varepsilon f(t)$, even only for sequences for which $v(n)=\frac{6 m+9}{2 m+6} \varepsilon r$, then that summary must use $\Omega\left(\frac{\log n}{\varepsilon} v(n)\right)$ bits of space.

ProOF. We construct a family of input sequences of length $n$ and variability $\frac{6 m+9}{2 m+6} \varepsilon r$. Choose sets of $r$ different indices $1 \ldots n$ so that there are choose $(n, r)$ such sets.

For each set $S$ we define an input sequence $f_{S}$. We define $f_{S}(0)=m$ and the rest of $f_{S}$ recursively: $f_{S}(t)=f_{S}(t-1)$ if $t$ is not in $S$, and $f_{S}(t)=(2 m+3)-f_{S}(t-1)$ if $t$ is in $S$. (That is, switch between $m$ and $m+3$.)

If $A$ and $B$ are two different sets, then $f_{A} \neq f_{B}$ : let $i$ be the smallest index that is in one and not the other; say $i$ is in $A$. Then $f_{A}(1 \ldots(i-1))=f_{B}(1 \ldots(i-1))$, but $f_{A}(i) \neq f_{A}(i-1)=f_{B}(i-1)=f_{B}(i)$

The variability of any $f_{S}$ is $\frac{6 m+9}{2 m+6} \varepsilon r$ : There are $r / 2$ changes from $m$ to $m+3$ and another $r / 2$ from $m+3$ to $m$. When we switch from $m$ to $m+3$, we get $\left|f^{\prime}(t) / f(t)\right|=3 /(m+3)$, and when we switch from $m+3$ to $m$, we get $\left|f^{\prime}(t) / f(t)\right|=3 / m$. Thus $\sum_{t}\left|\frac{f^{\prime}(t)}{f(t)}\right|=\frac{r}{2} \frac{6 m+9}{m(m+3)}=\frac{6 m+9}{2 m+6} \varepsilon r$.

There are choose $(n, r) \geq(n / r)^{r}$ input sequences in our family, so to distinguish between any two input sequences we need at least $r \log (n / r)=\Omega(r \log n)$ bits. Any summary that can determine for each $t$ the value $f(t)$ to within $\pm \varepsilon f(t)$, must also distinguish between $f(t)=m$ and $f(t)=m+3$, since there is no value within $\varepsilon m$ of $m$ that is also within $\varepsilon(m+3)$ of $m+3$. Since this summary must distinguish between $f(t)=m$ and $f(t)=m+3$ for all $t$, it must distinguish between any two input sequences in the family, and therefore needs $\Omega(r \log n)$ bits.

\section{G. RANDOMIZED LOWER BOUND, LEMMA 4.3}

Lemma G.1. Let $\mathcal{F}$ be a family of sequences of length $n$ and variabilities $\leq v$ such that no two sequences in $\mathcal{F}$ match. If a summary $S(f)$ guarantees for all $f$ in $\mathcal{F}$ and all $t \leq n$ that $P(|f(t)-\hat{f}(t)| \leq \varepsilon f(t)) \geq 99 / 100$, then that summary must use $\Omega(\log |\mathcal{F}|)$ bits of space.

Proof. Let $S(f)$ be the summary for a sequence $f$, and sample $\hat{f}(1) \ldots \hat{f}(n)$ once each using $S(f)$ to get $\hat{f}$. Let $A$ be the event that $|\{t:|f(t)-\hat{f}(t)| \leq \varepsilon f(t)\}| \geq \frac{90}{100} n$. By Markov's inequality and the guarantee in the premise, we must have $P(A) \geq 9 / 10$.

Let $\omega$ define the random bits used in constructing $S(f)$ and in sampling $\hat{f}$. For any choice $\omega$ in $A$ we have that $\hat{f}$ overlaps with $f$ in at least $\frac{9}{10} n$ positions, which means that $\hat{f}$ overlaps with any other $g \in \mathcal{F}$ in at most $\frac{7}{10} n$ positions: at most the $\frac{6}{10} n$ in which $f$ and $g$ could overlap, plus the $\frac{1}{10} n$ in which $\hat{f}$ and $f$ might not overlap.

Define $F \subseteq \mathcal{F}$ to be the sequences $g$ that overlap with $\hat{f}$ in at least $\frac{9}{10} n$ positions. This means that when $\omega \in A$ we have $|F|=1$, and therefore with probability at least $9 / 10$ we can identify which sequence $f$ had been used to construct $S(f)$.

We now prove our claim by reducing the Index ${ }_{N}$ problem to the problem of tracing the history of a sequence $f$. The following statement of Index ${ }_{N}$ is roughly as in Kushilevitz and Nisan [9]. There are two parties, Alice and Bob. Alice has an input string $x$ of length $N=\log _{2}|\mathcal{F}|$ and Bob has an input string $i$ of length $\log _{2} N$ that is interpreted as an index into $x$. Alice sends a message to Bob, and then Bob must output $x_{i}$ correctly with probability at least $9 / 10$.

Consider the following algorithm for solving Index $N$. Alice deterministically generates a family $\mathcal{F}$ of sequences of length $n$ and variabilities $\leq v$ such that no two match, by iterating over all possible sequences and choosing each next one that doesn't match any already chosen. Her $\log _{2}|\mathcal{F}|$ bits of input $x$ index a sequence $f$ in $\mathcal{F}$. Alice computes a summary $S(f)$ and sends it to Bob. After receiving $S(f)$, Bob computes $\hat{f}(t)$ for every $t=1 \ldots n$, to get a sequence $\hat{f}$. He then generates $\mathcal{F}$ himself and creates a set $F$ of all sequences in $\mathcal{F}$ that overlap with $\hat{f}$ in at least $\frac{9}{10} n$ positions. If $F=\{f\}$, which it is with probability at least $9 / 10$, then Bob can infer every bit of $x$.

Since the $\operatorname{Index}_{N}$ problem is known to have a one-way communication complexity of $\Omega(N)$, it must be the case that $|S(f)|=\Omega(\log |\mathcal{F}|)$. 


\section{H. RANDOMIZED LOWER BOUND, LEMMA 4.4}

Lemma H.1. For all $\varepsilon \leq 1 / 2$, all $v \geq 32400 \varepsilon \ln C$, and all $n>3 v / \varepsilon$, there is a family $\mathcal{F}$ of size $e^{\Omega(v / \varepsilon)}$ of sequences of size $n$ such that (1) no two sequences match and (2) every sequence has variability at most $v$.

Proof. We construct $\mathcal{F}$ so that each of the two conditions holds (separately) with probability at least $4 / 5$. Let $m=1 / \varepsilon$. To construct one sequence in $\mathcal{F}$, first define $f(0)=m$ with probability $1 / 2$, else $f(0)=m+3$. Then, for $t=1 \ldots n$ : define $f(t)=(2 m+3)-f(t-1)$ with probability $p=v / 6 \varepsilon n$, else $f(t)=f(t-1)$. That is, switch from $m$ to $m+3$ (or vice-versa) with probability $p=v / 6 \varepsilon n$.

We first prove that the probability is at most $1 / 5$ that any two sequences $f$ and $g$ match. We have that

$$
P(f(0)=g(0))=1 / 2
$$

If at any point in time we have $f(t)=g(t)$, then

$$
\begin{aligned}
P(f(t+1)=g(t+1)) & =1-2 p(1-p)=: \alpha \\
\text { and } P(f(t+1) \neq g(t+1)) & =2 p(1-p)=1-\alpha
\end{aligned}
$$

Similarly, if $f(t) \neq g(t)$, then

$$
\begin{aligned}
P(f(t+1)=g(t+1)) & =1-\alpha \\
\text { and } P(f(t+1) \neq g(t+1)) & =\alpha
\end{aligned}
$$

The overlap between $f$ and $g$ is the number of times $t$ that $f(t)=g(t)$. We model this situation with a Markov chain $M$ with two states, $c$ for "same" (that is, $f=g$ ) and $d$ for "different" $(f \neq g)$. Let $s_{t}$ be the state after $t$ steps, and let $p_{t}=\left(p_{t}(c), p_{t}(d)\right)$ be the probabilities that $M$ is in state $c$ and $d$ after step $t$. The stationary distribution $\pi=$ $(1 / 2,1 / 2)$, which also happens to be our initial distribution. We can model the overlap between $f$ and $g$ by defining a function $y\left(s_{t}\right)=1$ if $s_{t}=c$ and $y\left(s_{t}\right)=0$ otherwise; then $Y=\sum_{t=1}^{n} y\left(s_{t}\right)$ is the overlap between $f$ and $g$.

The expected value $E(y(\pi))$ of $y$ evaluated on $\pi$ is $1 / 2$. The (1/8)-mixing time $T$ is defined as the smallest time $T$ such that $\frac{1}{2}\left\|M^{t} r_{0}-\pi\right\|_{1} \leq 1 / 8$ over all initial distributions $r_{0}$. Let $r_{0}$ be any initial distribution and $r_{t}=M^{t} r_{0}$. If we define $\Delta_{t}=r_{t}(c)-\pi(c)$, then $\Delta_{t}=(2 \alpha-1)^{t} \Delta_{0}$. We can similarly bound $\left|r_{t}(d)-\pi(d)\right|$, so we can bound

$$
\begin{aligned}
T & \leq \frac{\ln (8)}{\ln (1 /(2 \alpha-1))} \leq \frac{3}{(1-(2 \alpha-1))} \leq \frac{3}{2 p(1-p)} \leq \frac{3}{2 p} \\
& =\frac{9 \varepsilon n}{v}
\end{aligned}
$$

since $1-p \geq 1 / 2$ and since $1 / \ln (1 / x) \leq 1 /(1-x)$ for $x$ in $(0,1)$. With this information we can now apply a sledgehammer of a result by Chung, Lam, Liu, and Mitzenmacher [2]. Our fact H.2 is their theorem 3.1, specialized a bit to our situation:

FACT H.2. Let $M$ be an ergodic Markov chain with state space $S$. Let $T$ be its (1/8)-mixing time. Let $\left(s_{1}, \ldots, s_{n}\right)$ denote an $n$-step random walk on $M$ starting from its stationary distribution $\pi$. Let $y$ be a weight function such that $E(y(\pi))=\mu$. Define the weight of $\left\{s_{t}\right\}$ by $Y=\sum_{t=1}^{n} y\left(s_{t}\right)$. Then there exists some universal constant $C$ such that for all $0<\delta<1$ we have $P(Y \geq(1+\delta) \mu n) \leq C \exp \left(-\delta^{2} \mu n / 72 T\right)$.
Specifically, this means that

$$
P\left(Y \geq \frac{6}{10} n\right) \leq C \exp (-v /(25 \cdot 72 \cdot 9 \cdot \varepsilon))
$$

Since $v \geq 32400 \varepsilon \ln C$, we can also write

$$
P\left(Y \geq \frac{6}{10} n\right) \leq \exp (-v / 32400 \varepsilon)
$$

If $|\mathcal{F}|=\frac{1}{5} \exp (v /(2 \cdot 32400 \varepsilon))$, then by the union bound, with probability at least $4 / 5$, no pair of sequences $f, g$ matches.

We also must prove that there are enough sequences with variability at most $v$. The change in variability due to a single switch from $m$ to $m+3$ (or vice-versa) is at most $3 / m=3 \varepsilon$. For any sequence $f$, let $U_{t}=1$ if $f$ switched at time $t$, else $U_{t}=0$. The expected number of switches is $v / 6 \varepsilon$; using a standard Chernoff bound,

$$
P\left(\sum_{t} U_{t} \geq 2 v / 6 \varepsilon\right) \leq \exp (-v / 18 \varepsilon) \leq 1 / 10
$$

Suppose we sample $N$ sequences and $B$ of them have more than $2 v / 6 \varepsilon$ switches. In expectation $E(B) \leq \frac{1}{10} N$ of them have too many switches. By Markov's inequality we have that $P(B \geq N / 2) \leq 1 / 5$, so we can toss out the fewer than $N / 2$ bad sequences. This gives us a final size of $\mathcal{F}$ of $\frac{1}{10} \exp (v /(2 \cdot 32400 \varepsilon))$.

\section{TRACKING ITEM FREQUENCIES, SECTION 5.1}

\section{Problem definition.}

The problem of tracking item frequencies is only slightly different than the counting problem we've considered so far. In this problem there is a universe $U$ of items and we maintain a dataset $D(t)$ that changes over time. At each new timestep $n$, either some item $\ell$ from $U$ is added to $D$, or some item $\ell$ from $D$ is removed. This update is told to a single site $i$; that is, site $i(n)$ receives an update $f_{\ell}^{\prime}(n)= \pm 1$.

The frequency $f_{\ell}(t)$ of item $\ell$ at time $t$ is the number of copies of $\ell$ that appear in $D(t)$. The first frequency moment $F_{1}(t)$ at time $t$ is the total number of items $|D(t)|$. The problem is to maintain estimates $\hat{f}_{\ell}(n)$ at the coordinator so that $P\left(\left|f_{\ell}(n)-\hat{f}_{\ell}(n)\right| \leq \varepsilon F_{1}(n)\right)$ is close to 1 , for all times $n$ and all items $\ell$.

Since in this problem we are tracking each item frequency to $\varepsilon F_{1}(n)$, we use $F_{1}$-variability instead, defining the change $v^{\prime}(t)=\min \left\{1,1 / F_{1}(t)\right\}$.

\section{I.1 Item frequencies with low communication}

We first partition time into blocks as in section 3.1, using $f=F_{1}$. That is, at the end of each block we know the values $n$ and $F_{1}(n)$ deterministically, and also that either $r=0$ holds or that $F_{1}\left(n_{j}\right)$ is within a factor of two of $F_{1}\left(n_{j-1}\right)$.

For tracking during blocks we modify the deterministic algorithm so that each site $i$ holds counters $d_{i \ell}$ and $\delta_{i \ell}$ for every item $\ell$. It also holds counters $f_{i \ell}$ of the total number of copies of $\ell$ seen at site $i$ across all blocks.

At the end of each block, each site $i$ reports all $f_{i \ell} \geq \varepsilon 2^{r} / 3$ (using the new value of $r$ ). If site $i$ reports counter $\bar{f}_{i \ell}$ then it starts the next block with $d_{i \ell}=\delta_{i \ell}=0$; otherwise, $d_{i \ell}$ is updated to $d_{i \ell}+\delta_{i \ell}$ and then $\delta_{i \ell}$ is reset to zero. Within a block $r \geq 1$, the CONDITION is true when $\delta_{i \ell} \geq \varepsilon 2^{r} / 3$.

The coordinator maintains estimates $\hat{f}_{i \ell}$ of $f_{i \ell}$ for each site $i$ and item $\ell$. Upon receiving an update $\delta_{i \ell}$ during a block the coordinator updates its estimate $\hat{f}_{i \ell}=\hat{f}_{i \ell}+\delta_{i \ell}$. 


\section{Estimation error.}

The total error in the estimate $\hat{f}_{i \ell}(n)$ at any time $n$ is the error due to $d_{i \ell}$ plus the error due to $\delta_{i \ell}$. In both cases these quantities are bounded by $\varepsilon 2^{r} / 3 \leq \varepsilon F_{1}(n) / 3$.

\section{Communication.}

The total communication for a block is the total communicated within and at the end of the block. Within a block, all $\delta_{i \ell}$ start at zero, and there are at most $2^{r} k$ updates, so the total number of messages sent is $3 k / \varepsilon$. At the end of a block, $f_{i \ell} \geq \varepsilon 2^{r} / 3$ is true for at most $12 k / \varepsilon$ counters $f_{i \ell}$. Therefore the total number of messages $O\left(\frac{k}{\varepsilon} v(n)\right)$.

\section{I.2 Item frequencies in small space + communication}

The algorithm so far uses $|U|$ counters per site, which is prohibitive in terms of space. In [3] Cormode and Muthukrishnan show that in order to track over a non-distributed update stream each $f_{\ell}(n)$ so that for all $\ell$ and all times $n$ we have $P\left(\left|f_{\ell}(n)-\hat{f}_{\ell}(n)\right| \leq \varepsilon F_{1}(n) / 3\right) \geq 8 / 9$, it suffices to randomly partition each item in $U$ into one of $27 / \varepsilon$ classes using a pairwise-independent hash function $h$, and to estimate $f_{\ell}(n)$ as $f_{h(\ell)}(n)$. The $27 / \varepsilon$ counters and the hash function $h$ together form their Count-Min Sketch [3].

Similarly, in [6, 7] Ganguly and Majumder adapt a data structure of Gasieniec and Muthukrishnan [12], which they call the CR-precis, to deterministically track each $f_{\ell}(n)$ to $\varepsilon F_{1}(n) / 3$ error. This data structure uses $\frac{3}{\varepsilon}$ rows of $\frac{6 \log |U|}{\varepsilon \log 1 / \varepsilon}$ counters, and estimates $f_{\ell}(n)$ as the average over rows $r$ of $f_{h(r, \ell)}(n)$. (Ganguly and Majumder actually take the minimum over the rows $r$, but the average works too and yields a linear sketch.)

In either case, we can first reduce our set of items $\ell$ to a small number of counters $c$, and instead of tracking $f_{i \ell}$ we track $f_{i c}$ for each counter $c$. The coordinator can then linearly combine its estimates $\hat{f}_{i c}$ to obtain estimates $\hat{f}_{i \ell}$ for each item $\ell$. This introduces another $\varepsilon F_{1}(n) / 3$ error, yielding algorithms that guarantee

$$
\text { - } \begin{array}{r}
P\left(\left|f_{i \ell}(n)-\hat{f}_{i \ell}(n)\right| \leq \varepsilon F_{1}(n)\right)=1 \text { in } \\
O\left(\frac{k \log |U|}{\varepsilon^{2} \log 1 / \varepsilon} v(n) \log n\right)
\end{array}
$$

bits of space + communication, and

$$
\begin{array}{r}
-P\left(\left|f_{i \ell}(n)-\hat{f}_{i \ell}(n)\right| \leq \varepsilon F_{1}(n)\right) \geq 8 / 9 \text { in } \\
O\left(k \log |U|+\frac{k}{\varepsilon} v(n) \log n\right)
\end{array}
$$

bits of space + communication.

\section{I.3 Remarks}

We obtain a randomized communication bound of $O\left(\frac{k}{\varepsilon} v(n)\right)$ messages, but it might be possible to do better. In [8] Huang et. al. both develop a randomized counting algorithm $\left(O\left(\frac{\sqrt{k}}{\varepsilon} \log n\right)\right.$ messages $)$ and also extend it to the problem of tracking item frequencies to get the same communication bound. Unfortunately, their algorithm appears to require the total variance in their estimate at any time $t<n$ to be bounded by a constant factor of the variance at time $n$. This is only guaranteed to be true when item deletions are not permitted (and $F_{1}$ grows monotonically). We avoid this problem in section 3.4 for tracking $f=F_{1}$ by deterministically updating $F_{1}$ at the end of each block. For this problem, though, deterministically updating all of the large $\hat{f}_{i \ell}$ at the end of each block could incur $O(k / \varepsilon)$ messages. Whether it is also possible to probabilistically track item frequencies over general update streams in $O\left(\frac{\sqrt{k}}{\varepsilon} v(n)\right)$ messages remains open.

\section{J. AGGREGATE FUNCTIONS WITH ONE SITE, SECTION 5.2}

The single-site algorithm of section 5.2 is: whenever we have $|f-\hat{f}|>\varepsilon f$ we send $f$ to the coordinator.

Proof. If $f(n)=0$ then $v^{\prime}(n)=1$. Also, if $f(n)$ changes sign from $f(n-1)$, then $v^{\prime}(n)=1$. So consider intervals over which $f(n)$ is nonzero and doesn't change sign. Over such an interval, let $\Phi(n)=\left|\frac{f(n)-\hat{f}(n)}{f(n)}\right|$. If at time $n$ we update $\hat{f}$ then $\Phi(n)=0$. Otherwise,

$$
\begin{aligned}
\Phi(n) & =\frac{\left|f(n-1)-\hat{f}(n-1)+f^{\prime}(n)\right|}{|f(n)|} \\
& \leq \frac{|f(n-1)-\hat{f}(n-1)|}{|f(n)|}+\frac{\left|f^{\prime}(n)\right|}{|f(n)|} \\
& =\frac{|f(n-1)|}{|f(n)|} \Phi(n-1)+\frac{\left|f^{\prime}(n)\right|}{|f(n)|} \\
& \leq \frac{|f(n)|+\left|f^{\prime}(n)\right|}{|f(n)|} \Phi(n-1)+\frac{\left|f^{\prime}(n)\right|}{|f(n)|} \\
& \leq \Phi(n-1)+\frac{(1+\Phi(n-1))\left|f^{\prime}(n)\right|}{|f(n)|}
\end{aligned}
$$

Since $\Phi(n) \leq \varepsilon$ we have $\left|\Phi^{\prime}(n)\right| \leq(1+\varepsilon)\left|\frac{f^{\prime}(n)}{f(n)}\right|$. We only send a message each time that $\Phi$ would be more than $\varepsilon$, so the total number of messages sent is at most the total increase in $\Phi$, which is at most $\frac{1+\varepsilon}{\varepsilon} \sum_{t=1}^{n} \min \left\{1,\left|\frac{f^{\prime}(t)}{f(t)}\right|\right\}$. 